\title{
Torsion of Anisotropic Elastic Cylinders by Forces Applied on the Lateral Surface ${ }^{1}$
}

\author{
Harold Luxenberg ${ }^{2}$
}

\begin{abstract}
The classical Saint-Venant theory of torsion presents a method for the determination of the elastic behavior of an orthotropic elastic beam of uniform arbitrary cross section twisted by forces applied on the end sections, with the lateral surface free of stress. In the present paper the theory is extended to include a more generally anisotropic beam (possessing only a single plane of elastic symmetry, rather than three mutually orthogonal planes of elastic symmetry, as in the case of orthotropic materials) built in at one end and twisted by forces uniformly distributed along the lateral surface.

The stresses in a beam of elliptic cross section twisted by constant tangential traction are obtained as an application of the theory
\end{abstract}

\section{Introduction}

The classical Saint-Venant theory of torsion presents a method for the determination of the elastic behavior of an orthotropic elastic beam of uniform arbitrary cross section twisted by forces applied on the end sections, with the lateral surface free of stress. In the present paper the theory is extended to include a more generally anisotropic cylinder (possessing only a single plane of elastic symmetry, rather than three mutually orthogonal planes of elastic symmetry, as in orthotropic materials) built in at one end and twisted by forces uniformly distributed along the lateral surface.

The extension of the Saint-Venant theory of torsion of cylinders by forces applied on the end sections to anisotropic cylinders was made by Lechnitzky $[3,4]^{3}$ in 1939 :

The torsion of isotropic cylinders by,forces applied on the lateral surface has been considered by Filon [1] in 1902, Timpe [11] in 1912, and Zwolinsky and Riz [12] in 1939.

Filon and Timpe showed that the stresses and displacements in an isotropic circular cylinder twisted by forces applied on the lateral surface are derivable from a biharmonic stress function. Zwolinsky and Riz treated the problem of the isotropic cylinder of arbitrary cross section twisted by constant tangential forces, and showed that, in this case, the stresses and displacements are derivable from two stress functions, one biharmonic, the other harmonic. For a circular cylinder the harmonic stress function vanishes, and the Filon-Timpe solution is obtained as a special case of the Zwolinsky-Riz solution.

The results obtained here are more general than those given by Zwolinsky and Riz in two important respects. First, the applied forces assumed in the present treatment are not necessarily constant or tangential to the lateral surface; and second, the results are applicable to a wider range of elastic materials, including wood, which is orthotropic, and

\footnotetext{
1 The preparation of this paper was sponsored (in part) by a grant from the Research Corporation of New York through the Regents of the University of California, and (in part) by the Office of Naval Research.

2;:Present address, Remington \& Rand, Inc., Philadelphia, Pa.

${ }_{3}$ Figures in brackets indicate the literature references at the end of this paper.
}

certain crystalline media [5, p. 155 to 160], which possess a single plane of elastic symmetry.

It will be noted that no mention has been made of the distribution of stresses over the built-in end of the cylinder. This distribution will, in general, depend on the mode of clamping. If these stresses are prescribed the exact solution of the problem presents considerable difficulty because of the complicated boundary conditions.

In 1855 Saint-Venant stated as a principle of elasticity that two statically equivalent systems of forces applied to a portion of the surface of a medium will produce essentially the same stresses at point of the body not too near the region of application of the forces.

If this principle is accepted, and if it is borne in mind that in most applications only the resultant of the stresses on the end of the cylinder is known, it is seen that a solution of practical usefulness may be obtained by "relaxing" the boundary conditions, and specifying only the resultant stress on the end section.

The extra freedom allowed by the relaxation of the boundary conditions permits us to obtain a solution by the so-called semi-inverse method of SaintVenant, which consists in making certain assumptions about the stress distributions, while still leaving enough arbitrariness in the assumed stresses to satisfy all the conditions of the problem.

$\mathrm{By}$ the use of this method it is shown that the stresses in the twisted cylinder are derivable from two stress functions, $\phi(x, y)$ and $\psi(x, y)$, which satisfy the differential equations.

$$
\left.\begin{array}{c}
l \frac{\partial^{2} \phi}{\partial x^{2}}+2 m \frac{\partial^{2} \phi}{\partial x \partial y}+n \frac{\partial^{2} \phi}{\partial y^{2}}=0, \\
b_{22} \frac{\partial^{4} \psi}{\partial x^{4}}-3 b_{26} \frac{\partial^{4} \psi}{\partial x^{3} \partial y}+2\left(b_{12}+b_{66}\right) \frac{\partial^{4} \psi}{\partial x^{2} \partial y^{2}}- \\
3 b_{16} \frac{\partial^{4} \psi}{\partial x \partial y^{3}}+b_{11} \frac{\partial^{4} \psi}{\partial y^{4}}=b_{2} \frac{\partial^{2} \phi}{\partial x^{2}}-2 b_{6} \frac{\partial^{2} \phi}{\partial x \partial y}+ \\
b_{1} \frac{\partial^{2} \phi}{\partial y^{2}}+b_{0}
\end{array}\right\}
$$


in the region $R$ corresponding to the interior of the cylinder, and the conditions

$$
\left.\begin{array}{r}
\left(l \frac{\partial \phi}{\partial x}+m \frac{\partial \phi}{\partial y}-l y+m x\right) \cos (x, \nu)+ \\
\left(m \frac{\partial \phi}{\partial x}+n \frac{\partial \phi}{\partial y}-m y+n x\right) \cos (y, \nu)=0 \\
\frac{d}{d s}\left(\frac{\partial \psi}{\partial y}\right)=\left(l \phi+\frac{m}{2} x^{2}-\frac{N}{\alpha}\right) \cos (x, \nu)+ \\
\left(\frac{n}{2} x^{2}-\frac{l}{2} y^{2}+m \phi+\frac{T}{\alpha}\right) \cos (y, \nu) \\
-\frac{d}{d s}\left(\frac{\partial \psi}{\partial x}\right)=\left(\frac{n}{2} x^{2}-\frac{l}{2} y^{2}+m \phi-\frac{T}{\alpha}\right) \cos (x, \nu)+ \\
\left(n \phi-\frac{m}{2} y^{2}-\frac{N}{\alpha}\right) \cos (y, \nu)
\end{array}\right\}
$$

on the bounding curve $C$ of each cross section. The parameters $l, m, n, b_{i}$, and $b_{i j}$ appearing in these equations are functions of the 13 elastic constants of the material, $N$ and $T$ are the normal and tangential components of the applied surface tractions per unit area, $\nu$ designates the unit normal vector to $C$, and $\alpha$ is given by

$$
\alpha=\frac{\int_{c}(x Y-y X) d s}{\int_{R}\left[n x^{2}-2 m x y+l y^{2}+x\left(m \frac{\partial \phi}{\partial x}+n \frac{\partial \phi}{\partial y}\right)-y\left(l \frac{\partial \phi}{\partial x}+m \frac{\partial \phi}{\partial y}\right)\right] d S} .
$$

In eq (3), $X$ and $Y$ are the $x$ and $y$ components of the applied surface traction, and the contour integral is the applied twisting moment per unit length. The surface integral is proportional to the torsional rigidity of the cylinder.

In the case of an isotropic beam the differential equations (1), simplify to

$$
\nabla^{2} \phi=0, \quad \nabla^{4} \psi=0
$$

the boundary conditions (2) become

$$
\begin{aligned}
& \left(\frac{\partial \phi}{\partial x}-y\right) \cos (x, \nu)+\left(\frac{\partial \phi}{\partial y}+x\right) \cos (y, \nu)=0 \\
& \frac{d}{d s}\left(\frac{\partial \psi}{\partial y}\right)=\left(\frac{\phi}{2}-\frac{N}{\alpha}\right) \cos (x, \nu)+ \\
& \left(\frac{x^{2}-y^{2}}{4}+\frac{T}{\alpha}\right) \cos (y, \nu) \\
& -\frac{d}{d s}\left(\frac{\partial \psi}{\partial x}\right)=\left(\frac{x^{2}-y^{2}}{4}-\frac{T}{\alpha}\right) \cos (x, \nu)+ \\
& \left(\frac{\phi}{2}-\frac{N}{\alpha}\right) \cos (y, \nu)
\end{aligned}
$$

and $\alpha$ is given by

$$
\alpha=2 \frac{\int_{C}(x Y-y X) d s}{\int_{R}\left(x^{2}+y^{2}+x \frac{\partial \phi}{\partial y}-y \frac{\partial \phi}{\partial x}\right) d S} .
$$

It is shown, from energy considerations, that the boundary-value problems posed above possess a unique solution, and several methods of solution are discussed.

As an illustration of the method, the stresses in a. cylinder of elliptic cross section twisted by constant, tangential traction are obtained.

\section{General theory}

The first fundamental boundary-value problem of elasticity may be stated as follows [8, p. 72]:

Determine the distribution of stress and the displacements in the interior of an elastic body in equilibrium when the body forces are prescribed and the distribution of the forces acting on the surface of the body is known.

The second fundamental problem is similar, except that in this case the surface displacements rather than the forces acting on the surface are prescribed, and in the third, or mixed, boundary-value problem the forces are prescribed over a portion of the surface and the displacements over the remainder.

The problem of the cylinder that we are considering is of the first kind, where body forces are absent.

In the following paragraphs we shall review certain basic elements of the mathematical theory of elasticity and present the mathematical formulation of the first fundamental boundary-value problem.

The state of stress in an elastic medium is characterized by the six components $\tau_{x x}, \tau_{y y}, \tau_{z z}, \tau_{y z}, \tau_{z x}$, $\tau_{x y}$, of the stress tensor. The components of stress, $X_{\nu}, Y_{\nu}, Z_{v}$, on an element of surface whose normal has the direction $\vec{\nu}$ are given in terms of the stress tensor by the relations [8, p. 40]

$$
\left.\begin{array}{l}
X_{\nu}=\tau_{x x} \cos (x, \nu)+\tau_{x y} \cos (y, \nu)+\tau_{z x} \cos (z, \nu) \\
Y_{\nu}=\tau_{x y} \cos (x, \nu)+\tau_{y y} \cos (y, \nu)+\tau_{y z} \cos (z, \nu) \\
Z_{\nu}=\tau_{z x} \cos (x, \nu)+\tau_{y z} \cos (y, \nu)+\tau_{z z} \cos (z, \nu) .
\end{array}\right\}
$$

The relations (7) may be regarded as defining the six components, $\tau_{x x}, \tau_{y y}, \ldots$. , $\tau_{x y}$, of the stress tensor. If we let the vector $\vec{\nu}$ take on the directions of the three coordinate axes in eq (7), we obtain the usual definition of the stress tensor,

$$
\left.\begin{array}{rll}
X_{x} \equiv \tau_{x x}, & Y_{x} \equiv \tau_{x y}, & Z_{x} \equiv \tau_{z x} \\
X_{y} \equiv \tau_{x y}, & Y_{y} \equiv \tau_{y y}, & Z_{y} \equiv \tau_{y z} \\
X_{z} \equiv \tau_{z x}, & Y_{z} \equiv \tau_{y z}, & Z_{z} \equiv \tau_{z z}
\end{array}\right\}
$$


From considerations of static equilibrium it can be shown that

$$
X_{y}=Y_{x}, \quad Z_{x}=X_{z}, \quad Y_{z}=Z_{y},
$$

and that the defining relations (8) are self-consistent.

For any material in static equilibrium under a prescribed system of surface loading $X_{\nu}, Y_{\nu}, Z_{\nu}$, and free of body forces, the stress tensor must satisfy the equilibrium equations

$$
\left.\begin{array}{l}
\frac{\partial \tau_{x x}}{\partial x}+\frac{\partial \tau_{x y}}{\partial y}+\frac{\partial \tau_{z x}}{\partial z}=0 \\
\frac{\partial \tau_{x y}}{\partial x}+\frac{\partial \tau_{y y}}{\partial y}+\frac{\partial \tau_{y z}}{\partial z}=0 \\
\frac{\partial \tau_{z x}}{\partial x}+\frac{\partial \tau_{y z}}{\partial y}+\frac{\partial \tau_{z z}}{\partial z}=0
\end{array}\right\}
$$

in the interior of the medium, and the conditions (7) on the surface, where we now regard $X_{\nu}, Y_{\nu}, Z_{\nu}$ as the prescribed surface tractions.

The strain tensor, $e_{x x}, e_{y y}, e_{z z}, e_{y z}, e_{z x}, \mathrm{e}_{x y}$, is defined in terms of $u, v$, and $w$, the $x, y$, and $z$ components of the displacement vector, by the relations

$$
\left.\begin{array}{l}
e_{x x}=\frac{\partial u}{\partial x}, \quad e_{y y}=\frac{\partial v}{\partial y}, \quad e_{z z}=\frac{\partial w}{\partial z}, \\
e_{y z}=\frac{1}{2}\left(\frac{\partial w}{\partial y}+\frac{\partial v}{\partial z}\right), \quad e_{z x}=\frac{1}{2}\left(\frac{\partial u}{\partial z}+\frac{\partial w}{\partial x}\right), \\
e_{x y}=\frac{1}{2}\left(\frac{\partial v}{\partial x}+\frac{\partial u}{\partial y}\right) .
\end{array}\right\}
$$

It may be shown [5, p. 48] that a necessary and sufficient condition that a given tensor may be considered as a strain tensor arising from actual physical displacements in an elastic material is that the tensor satisfy the conditions

$$
\begin{aligned}
\frac{\partial^{2} e_{x x}}{\partial y \partial z} & =\frac{\partial}{\partial x}\left(-\frac{\partial e_{y z}}{\partial x}+\frac{\partial e_{z x}}{\partial y}+\frac{\partial e_{x y}}{\partial z}\right) \\
\frac{\partial^{2} e_{y y}}{\partial z \partial x} & =\frac{\partial}{\partial y}\left(-\frac{\partial e_{z x}}{\partial y}+\frac{\partial e_{x y}}{\partial z}+\frac{\partial e_{y z}}{\partial x}\right) \\
\frac{\partial^{2} e_{z z}}{\partial x \partial y} & =\frac{\partial}{\partial z}\left(-\frac{\partial^{2} e_{x y}}{\partial z}+\frac{\partial^{2} e_{y z}}{\partial x}+\frac{\partial e_{z x}}{\partial y}\right) \\
2 \frac{\partial^{2} e_{x y}}{\partial x \partial y} & =\frac{\partial^{2} e_{x x}}{\partial y^{2}}+\frac{\partial^{2} e_{y y}}{\partial x^{2}} \\
2 \frac{\partial^{2} e_{y z}}{\partial y \partial z} & =\frac{\partial^{2} e_{y y}}{\partial z^{2}}+\frac{\partial^{2} e_{z z}}{\partial y^{2}} \\
2 \frac{\partial^{2} e_{z z}}{\partial z \partial x} & =\frac{\partial^{2} e_{z z}}{\partial x^{2}}+\frac{\partial^{2} e_{x x}}{\partial z^{2}} .
\end{aligned}
$$

Equations (12) are known as the compatibility equations and are the integrability conditions for the system of (11), when the displacements $u, v, w$ are to be determined from a given strain tensor.

The stress and strain tensors in an elastic medium are related by the generalized Hooke's law. If the elastic constants of the medium are denoted by $c_{i j}(i, j=1,2, \ldots, 6)$ Hooke's law for a medium in which the $x y$-plane is a plane of elastic symmetry ${ }^{4}$ may be written in the form [8, p. 62]:

$$
\left.\begin{array}{l}
e_{x x}=c_{11} \tau_{x x}+c_{12} \tau_{y y}+c_{13} \tau_{z z}+c_{16} \tau_{x y} \\
e_{y y}=c_{21} \tau_{x x}+c_{22} \tau_{y y}+c_{23} \tau_{z z}+c_{26} \tau_{x y} \\
e_{z z}=c_{31} \tau_{x x}+c_{32} \tau_{y y}+c_{33} \tau_{22}+c_{36} \tau_{x y} \\
e_{y z}=c_{44} \tau_{y z}+c_{45} \tau_{z x} \\
e_{z x}=c_{54} \tau_{y z}+c_{55} \tau_{z x} \\
e_{x y}=c_{61} \tau_{x x}+c_{62} \tau_{y y}+c_{63} \tau_{z z}+c_{66} \tau_{x y}
\end{array}\right\},
$$

where $c_{12}=c_{21}, \quad c_{13}=c_{31}, \quad c_{16}=c_{61}, \quad c_{23}=c_{32}, \quad c_{26}=c_{62}$, $c_{36}=c_{63}, c_{45}=c_{54}$.

For isotropic materials, the number of independent elastic constants reduces to two, and Hooke's law has the simpler form

$$
\left.\begin{array}{c}
e_{x x}=\frac{1}{E} \tau_{x x}-\frac{\sigma}{E}\left(\tau_{y y}+\tau_{z z}\right), \quad e_{y y}=\frac{1}{E} \tau_{y y}- \\
\frac{\sigma}{E}\left(\tau_{z z}+\tau_{x x}\right), \quad e_{z z}=\frac{1}{E} \tau_{z z}-\frac{\sigma}{E}\left(\tau_{x x}+\tau_{y y}\right), \\
e_{y z}=\frac{1+\sigma}{E} \tau_{y z}, \quad e_{z x}=\frac{1+\sigma}{E} \tau_{z x}, \quad e_{x y}=\frac{1+\sigma}{E} \tau_{x y},
\end{array}\right\}
$$

where $E$ is Young's modulus and $\sigma$ is Poisson's ratio for the material.

We may now reformulate the first fundamental boundary-value problem of elasticity in mathematical terms:

Determine a set of six functions, the components of the stress tensor, which satisfies the equations (10) in the interior of the elastic medium and the relations (7) on the surface of the medium, where $X_{\nu}, Y_{\nu}$, and $Z_{\nu}$ are prescribed functions, and such that the strain tensor calculated from these functions by the use of (13) satisfies the compatibility conditions (12).

\section{Formulation of the problem}

Let the coordinate system be chosen as shown in figure 1, with the built-in end of the cylinder in the

4 The most general elastic medium is characterized by 21 independent elastic constants. Here we consider a medium whose elastic properties are identical in constants. Here we consider a medium whose elastic properties are identical in any two directions symmetric with respect to the $x y$-plane. This type
symmetry reduces the number of independent elastic constants to 13 . 
plane $z=0$, and the free end in the plane $z=h$. The boundary curve of any cross section will be denoted by $C$; the region enclosed by $C$ and the unit normal to $C$ will be denoted by $R$ and $\vec{\nu}$.
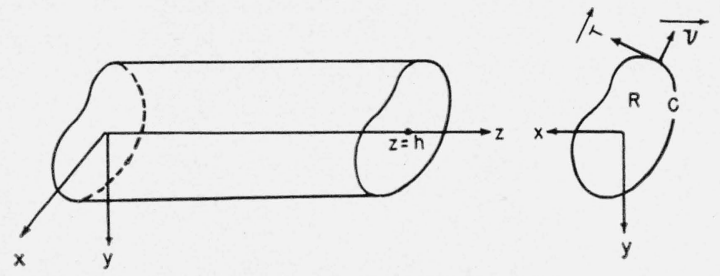

FIGURE 1.

The prescribed surface traction $\vec{T}$ (force per unit area) is assumed to be independent of $z$, directed parallel to the $(x, y)$ plane, and such that the resultant on any cross section is a twisting moment of magnitude $M$ (torque per unit length).

This condition requires that the components $X$ and $Y$ of the applied surface traction satisfy the relations

$\int_{C} X d s=0, \quad \int_{C} Y d s=0, \quad \int_{C}(x Y-y X) d s=M$.

In order that the first fundamental boundary value problem of elasticity may be formulated in such a way that the solution is unique, it is necessary that the stresses be prescribed over the entire surface, subject, of course, to the conditions of static equilibrium. This would require that $Z_{x}$ and $Z_{y}$, the components of the clamping stresses on the end $z=0$, be prescribed, subject to the conditions

$\int_{R} Z_{x} d S=0, \quad \int_{R} Z_{y} d S=0, \quad \int_{R}\left(x Z_{y}-y Z_{x}\right) d S=M h$.

In this case the boundary conditions on the end sections would be of the form:

(a) on the end $z=0$ :

$$
\tau_{z z}=0, \quad \tau_{z x}=Z_{x}, \quad \tau_{y z}=Z_{y}
$$

(b) on the end $z=h$ :

$$
\tau_{z z}=0, \quad \tau_{z x}=0, \quad \tau_{y z}=0 .
$$

Inasmuch as $Z_{x}$ and $Z_{y}$ are unknown (and depend upon the mode of clamping), we shall replace the exact boundary conditions (16) by the relaxed conditions, (a) on the end $z=0$ :

$$
\begin{aligned}
& \int_{R} \tau_{z z} d S=\int_{R} x \tau_{z z} d S=\int_{R} y \tau_{z z} d S=0 \\
& \int_{R} \tau_{z x} d S=\int_{R} \tau_{y z} d S=0 \\
& \int_{R}\left(x \tau_{z y}-y \tau_{z x}\right) d S=M h
\end{aligned}
$$

(b) on the end $z=h$ :

$$
\begin{gathered}
\int_{R} \tau_{z z} d S=\int_{R} x \tau_{z z} d S=\int_{R} y \tau_{z z} d S=0 \\
\tau_{z x}=0 \\
\tau_{y z}=0 .
\end{gathered}
$$

These are conditions on the resultant stresses, rather than on the stress distributions.

The boundary conditions on the lateral surface, that is, on the curve $C$, are

$$
\left.\begin{array}{r}
\tau_{x x} \cos (x, \nu)+\tau_{x y} \cos (y, \nu)=X \\
\tau_{x y} \cos (x, \nu)+\tau_{y y} \cos (y, \nu)=Y \\
\tau_{z x} \cos (x, \nu)+\tau_{y z} \cos (y, \nu)=0 .
\end{array}\right\}
$$

Our problem, then, is to determine a system of stresses $\tau_{x x}, \tau_{y y}, \ldots, \tau_{x y}$ which satisfies the equilibrium eq (10), the relaxed boundary conditions (16a) and (17), and for which the strain system calculated by the use of Hooke's law (13) satisfies the compatibility equation (12).

The solution of the problem as thus formulated is, of course, not unique, but, according to the principle of Saint-Venant [8, p. 95], the stresses given by any two solutions will be essentially the same at points of the cylinder not too near the end sections.

We shall make use of the Saint-Venant semi-inverse method of solution [8, p. 100]. This consists in making certain assumptions about the stress distribution, and still leaving sufficient freedom to permit the satisfaction of the differential equations and the boundary conditions. If these assumptions are carefully made, a unique solution (unique under these assumptions) exists.

It is apparent that the torsional couple transmitted across each cross section is a linear function of the distance from that cross section to the free end of the cylinder. Hence, it is natural to assume that the stresses in the cylinder are given, in part at least, by a Saint-Venant torsion function.

There is a type of elastic deformation known as plane strain $[7$, p. 231], in which the displacements are of the form

$$
u=u(x, y), \quad v=v(x, y), \quad w \equiv 0 .
$$


The stresses in this type of deformation are derivable from an Airy stress function and can be chosen to satisfy very general boundary conditions of the type given by (17).

We shall assume that the stress system in the twisted cylinder may be derived, in part, from a superposition of the torsion. solution and the plane strain type solution.

For a deformation to be of plane strain type, it is necessary that $\tau_{z z} \neq 0$. Because a variable tension on the end sections may produce a bending or extension of the cylinder, we shall further superpose the bending and tension type solutions.

Thus, if we denote by $\tau_{i j}$ any one of the six components of the stress tensor, we may write

$$
\frac{\tau_{i j}}{\alpha}=\tau_{i j}^{(1)}+\tau_{i j}^{(2)}+\tau_{i j}^{(3)}
$$

where $\alpha$ is a constant to be determined by conditions of static equilibrium, $\tau_{i j}^{(1)}$ is derived from an Airy stress function, $\tau_{i j}^{(2)}$ is derived from the Saint-Venant torsion function, and $\tau_{i j}^{(3)}$ is required to satisfy the condition that the end surfaces be free of stresses that would produce bending moments and extensions.

The stress system $\tau_{i j}^{(1)}$ may be written [7, p. 231]

$$
\left.\begin{array}{c}
\tau_{x x}^{(1)}=-\frac{\partial^{2} \psi}{\partial x^{2}}, \quad \tau_{y y}^{(1)}=-\frac{\partial^{2} \psi}{\partial y^{2}} \\
\tau_{x y}^{(1)}=\frac{\partial^{2} \psi}{\partial x \partial y}, \quad \tau_{x z}^{(1)}=0, \quad \tau_{y z}^{(1)}=0 \\
\tau_{s z}^{(1)}=p \frac{\partial^{2} \psi}{\partial x^{2}}+q \frac{\partial^{2} \psi}{\partial x \partial y}+r \frac{\partial^{2} \psi}{\partial y^{2}},
\end{array}\right\}
$$

where $\psi[\equiv \psi(x, y)]$ is the Airy stress function and $p$, $q$, and $r$, are functions of the elastic constants. We note that the stress system $\tau_{i j}^{(1)}$ satisfies the equilibrium equation (10) identically.

The stress system $\tau_{i i}^{(3)}$ has the form [8, p. 101, 108]

$$
\tau_{s z}^{(3)}=A+B x+C y, \quad \tau_{x x}^{(3)}=\tau_{y y}^{(3)}=\tau_{x y}^{(3)}=\tau_{x z}^{(3)}=\tau_{y z}^{(3)}=0,
$$

where $A, B, C$ are determined by the three relations

$$
\int_{R} \tau_{z z} d S=0, \quad-\int_{R} x \tau_{z z} d S=0, \quad \int_{R} y \tau_{z z} d S=0
$$

which state that the resultant bending couples and tension vanish on the end sections.

The system $\tau_{i j}^{(2)}$ is a modification of the SaintVenant stress system $\tau_{i j}^{\prime}$. Sokolnikoff $[8$, p. 214] gives the stresses $\tau_{i j}^{\prime}$ in an orthotropic beam twisted by end couples as

$$
\left.\begin{array}{l}
\tau_{y z}^{\prime}=n\left(\frac{\partial \phi}{\partial y}+x\right)+m\left(\frac{\partial \phi}{\partial x}-y\right) \\
\tau_{z x}^{\prime}=l\left(\frac{\partial \phi}{\partial x}-y\right)+m\left(\frac{\partial \phi}{\partial y}+x\right) \\
\tau_{x x}^{\prime}=\tau_{y y}^{\prime}=\tau_{z z}^{\prime}=\tau_{x y}^{\prime}=0,
\end{array}\right\}
$$

where $l, m, n$ are functions of the elastic constants and $\phi[\equiv \phi(x, y)]$ is the torsion function. Because we wish the torsional couple to vanish on the face $z=h$, we shall set

$$
\left.\begin{array}{l}
\tau_{y z}^{(2)}=(h-z)\left\{n\left(\frac{\partial \phi}{\partial y}+x\right)+m\left(\frac{\partial \phi}{\partial x}-y\right)\right\} \\
\tau_{z x}^{(2)}=(h-z)\left\{l\left(\frac{\partial \phi}{\phi x}-y\right)+m\left(\frac{\partial \phi}{\partial y}+x\right)\right\} \cdot
\end{array}\right\}
$$

The third of the equilibrium equations (10) will be satisfied if $\phi(x, y)$ satisfies the condition

$$
l \frac{\partial^{2} \phi}{\partial x^{2}}+2 m \frac{\partial^{2} \phi}{\partial x \partial y}+n \frac{\partial^{2} \phi}{\partial y^{2}}=0 .
$$

In order that the first two equilibrium equations in the set (10) be satisfied identically, we set

$$
\begin{aligned}
\tau_{x x}^{(2)}=l \phi+\frac{m}{2} x^{2}, \quad \tau_{y y}^{(2)}= & n \phi-\frac{m}{2} y^{2}, \\
\tau_{x y}^{(2)} & =\frac{n}{2} x^{2}-\frac{l}{2} y^{2}+m \phi .
\end{aligned}
$$

To simplify the satisfaction of the compatibility conditions (12), we choose

$$
\tau_{x x}^{(2)}=-\phi+\frac{s}{2} x^{2}-\frac{t}{2} y^{2},
$$

with $s$ and $t$ as yet undetermined.

Our assumed stress system, then, is the following:

$$
\frac{1}{\alpha} \tau_{x x}=l \phi-\frac{\partial^{2} \psi}{\partial y^{2}}+\frac{m}{2} x^{2}
$$

$$
\begin{aligned}
& \frac{1}{\alpha} \tau_{y y}=n \phi-\frac{\partial^{2} \psi}{\partial x^{2}}-\frac{m}{2} y^{2} \\
& \frac{1}{\alpha} \tau_{z z}=p \frac{\partial^{2} \psi}{\partial x^{2}}+q \frac{\partial^{2} \psi}{\partial x \partial y}+r \frac{\partial^{2} \psi}{\partial y^{2}}-\phi+\frac{s}{2} x^{2}-\frac{t}{2} y^{2}+ \\
& A+B x+C y \\
& \frac{1}{\alpha} \tau_{y z}=(h-z)\left\{n\left(\frac{\partial \phi}{\partial y}+x\right)+m\left(\frac{\partial \phi}{\partial x}-y\right)\right\} \\
& \frac{1}{\alpha} \tau_{z x}=(h-z)\left\{l\left(\frac{\partial \phi}{\partial x}-y\right)+m\left(\frac{\partial \phi}{\partial y}+x\right)\right\} \\
& \frac{1}{\alpha} \tau_{z x}=\frac{n}{2} x^{2}-\frac{l}{2} y^{2}+\frac{\partial^{2} \psi}{\partial x \partial y}+m \phi
\end{aligned}
$$


with the constants $\alpha, l, m, n, p, q, r, s$, and $t$ as yet undetermined.

We next determine $p, q, r, s$, and $t$, in such a way that $e_{z z}$ will be independent of $\psi(x, y)$ and of the terms in $x^{2}$ and $y^{2}$. The insertion of the stresses given by (27) into the third of (13) leads to the following expression for $e_{z z}$ :

$$
\begin{aligned}
\frac{1}{\alpha} e_{z z}= & \left(c_{31} l+c_{32} n-c_{33}+c_{36} m\right) \phi+ \\
& c_{33}(A+B x+C y)+ \\
& \frac{1}{2}\left(c_{31} m+c_{33} s+c_{36} n\right) x^{2}- \\
& \frac{1}{2}\left(c_{32} m+c_{33} t+c_{36} l\right) y^{2}+\left(c_{33} p-c_{32}\right) \frac{\partial^{2} \psi}{\partial x^{2}}+ \\
& \left(c_{32} q+c_{36}\right) \frac{\partial^{2} \psi}{\partial x \partial y}+\left(c_{33} r-c_{31}\right) \frac{\partial^{2} \psi}{\partial y^{2}} .
\end{aligned}
$$

If we set

$$
\begin{gathered}
p=c_{32} / c_{33}, \quad q=-c_{36} / c_{33}, \quad r=c_{31} / c_{33}, \\
s=-\left(c_{31} m+c_{36} n\right) / c_{33}, \quad t=-\left(c_{32} m+c_{36} l\right) / c_{33},
\end{gathered}
$$

the last five terms in the right-hand member of (28) will vanish.

If the values for $p, q, r, s$, and $t$ given in (29) are substituted in the stress equations (27), and the strains are then calculated from Hooke's law (13), we have the following expressions for the components of the strain tensor:

$$
\begin{aligned}
\frac{1}{\alpha} e_{x x}= & b_{1} \phi-b_{12} \frac{\partial^{2} \psi}{\partial x^{2}}+b_{16} \frac{\partial^{2} \psi}{\partial x \partial y}-b_{11} \frac{\partial^{2} \psi}{\partial y^{2}}+ \\
& \left(b_{11} m+b_{16} n\right) \frac{x^{2}}{2}-\left(b_{16} l+b_{12} m\right) \frac{y^{2}}{2}+ \\
& c_{13}(A+B x+C y) \\
\frac{1}{\alpha} e_{y y}= & b_{2} \phi-b_{22} \frac{\partial^{2} \psi}{\partial x^{2}}+b_{26} \frac{\partial^{2} \psi}{\partial x \partial y}-b_{21} \frac{\partial^{2} \psi}{\partial y^{2}}+ \\
& \left(b_{21} m+b_{26} n\right) \frac{x^{2}}{2}-\left(b_{26} l+b_{22} m\right) \frac{y^{2}}{2}+ \\
& c_{23}(A+B x+C y) \\
\frac{1}{\alpha} e_{z z}= & b_{3} \phi+c_{33}(A+B x+C y) \\
\frac{1}{\alpha} e_{y z}= & (h-z)\left\{\left(c_{44} n+c_{45} m\right)\left(\frac{\partial \phi}{\partial y}+x\right)+\right. \\
& \left.\left(c_{44} m+c_{45} l\right)\left(\frac{\partial \phi}{\partial x}-y\right)\right\} \\
\frac{1}{\alpha} e_{z x}= & (h-z)\left\{\left(c_{54} m+c_{55} l\right)\left(\frac{\partial \phi}{\partial x}-y\right)+\right. \\
& \left.\left(c_{54} n+c_{55} m\right)\left(\frac{\partial \phi}{\partial y}+x\right)\right\}
\end{aligned}
$$

$$
\begin{aligned}
\frac{1}{\alpha} e_{x y}= & b_{6} \phi-b_{62} \frac{\partial^{2} \psi}{\partial x^{2}}+b_{66} \frac{\partial^{2} \psi}{\partial x \partial y}-b_{61} \frac{\partial^{2} \psi}{\partial y^{2}}+ \\
& \left(b_{61} m+b_{66} n\right) \frac{x^{2}}{2}-\left(b_{66} l+b_{62} m\right) \frac{y^{2}}{2}+ \\
& c_{63}(A+B x+C y),
\end{aligned}
$$

where

$$
\left.\begin{array}{c}
b_{i j}=b_{j i}=\left|\begin{array}{l}
c_{i j} c_{i 3} \\
c_{3 j} c_{33}
\end{array}\right| / c_{33}, \quad i, j=1,2,6 \\
b_{i}=c_{i 1} l+c_{i 2} n-c_{i 3}+c_{26} m, \quad i=1,2,3,6 .
\end{array}\right\}
$$

If the components of the train tensor given by (30) are inserted in the compatibility equations (12), it is seen that all but the fourth of the compatibility equations are satisfied by the assumed stresses if $l$, $m$, and $n$ can be chosen to satisfy the six equations

$$
\left.\begin{array}{c}
c_{44} n-c_{55} l=0 \\
c_{45} l+c_{44} m=0 \\
c_{45} n+c_{55} m=0 \\
l\left(c_{55}+c_{31}\right)+m\left(2 c_{45}+c_{36}\right)+n\left(c_{44}+c_{32}\right)=c_{33} \\
l\left(c_{31}\right)+m\left(2 c_{45}+c_{36}\right)+n\left(2 c_{44}+c_{32}\right)=c_{33} \\
l\left(2 c_{55}+c_{31}\right)+m\left(2 c_{45}+c_{36}\right)+n\left(c_{32}\right)=c_{33} .
\end{array}\right\}
$$

It is easily seen that only three of these six equations are linearly independent and that the compatibility equations are satisfied if we set

$$
l=c_{33} c_{44} / \beta, \quad m=-c_{33} c_{45} / \beta, \quad n=c_{33} c_{55} / \beta,
$$

where

$$
\beta=c_{44}\left(c_{55}+c_{31}\right)+c_{55}\left(c_{44}+c_{32}\right)-c_{45}\left(2 c_{45}+c_{36}\right) .
$$

For convenience in calculation we rewrite the expressions for $s$ and $t$ given by (29) in terms of the elastic constants, using the relations (33):

$$
s=\left(c_{31} c_{45}-c_{36} c_{55}\right) / \beta, \quad t=\left(c_{32} c_{45}-c_{36} c_{44}\right) / \beta .
$$

The fourth of the compatibility equations (12), the only one that is dependent on $\psi(x, y)$, requires that $\psi(x, y)$ satisfy the relation

$$
\begin{array}{r}
b_{22} \frac{\partial^{4} \psi}{\partial x^{4}}-3 b_{26} \frac{\partial^{4} \psi}{\partial x^{3} \partial y}+2\left(b_{12}+b_{66}\right) \frac{\partial^{4} \psi}{\partial x^{2} \partial y^{2}}- \\
3 b_{16} \frac{\partial^{4} \psi}{\partial x \partial y^{3}}+b_{11} \frac{\partial^{4} \psi}{\partial y^{4}}=b_{2} \frac{\partial^{2} \phi}{\partial x^{2}}-2 b_{6} \frac{\partial^{2} \phi}{\partial x \partial y}+ \\
b_{1} \frac{\partial^{2} \phi}{\partial y^{2}}+b_{26} n-b_{16} l .
\end{array}
$$

In the next section we shall consider the boundary conditions on the functions $\phi(x, y)$ and $\psi(x, y)$. 


\section{Boundary conditions}

It has been shown in the previous section that the system of stresses given by

$$
\begin{aligned}
\frac{1}{\alpha} \tau_{x x}= & l \phi-\frac{\partial^{2} \psi}{\partial y^{2}}+\frac{m}{2} x^{2} \\
\frac{1}{\alpha} \tau_{y y}= & n \phi-\frac{\partial^{2} \psi}{\partial x^{2}}-\frac{m}{2} y^{2} \\
\frac{1}{\alpha} \tau_{z z}= & \frac{c_{32}}{c_{33}} \frac{\partial^{2} \psi}{\partial x^{2}}-\frac{c_{36}}{c_{33}} \frac{\partial^{2} \psi}{\partial x \partial y}+\frac{c_{31}}{c_{33}} \frac{\partial^{2} \psi}{\partial y^{2}}+ \\
& \frac{c_{31} c_{45}-c_{36} c_{55}}{2 \beta} x^{2}-\frac{c_{32} c_{45}-c_{36} c_{44}}{2 \beta} y^{2}-\phi+ \\
& A+B x+C y
\end{aligned}
$$$$
\frac{1}{\alpha} \tau_{y z}=(h-z)\left\{n\left(\frac{\partial \phi}{\partial y}+x\right)+m\left(\frac{\partial \phi}{\partial x}-y\right)\right\}
$$$$
\frac{1}{\alpha} \tau_{z x}=(h-z)\left\{l\left(\frac{\partial \phi}{\partial x}-y\right)+m\left(\frac{\partial \phi}{\partial y}+x\right)\right\}
$$$$
\frac{1}{\alpha} \tau_{x y}=\frac{n}{2} x^{2}-\frac{l}{2} y^{2}+\frac{\partial^{2} \psi}{\partial x \partial y}+m \phi,
$$

will satisfy the equilibrium equations (10) if $\phi(x, y)$ satisfies

$$
l \frac{\partial^{2} \phi}{\partial x^{2}}+2 m \frac{\partial^{2} \phi}{\partial x \partial y}+n \frac{\partial^{2} \phi}{\partial y^{2}}=0,
$$

and will satisfy the compatibility conditions (12), provided that

$$
\left.\begin{array}{l}
l=c_{33} c_{44} / \beta, \quad m=-c_{33} c_{45} / \beta, \quad n=c_{33} c_{55} / \beta \\
\beta=c_{44}\left(c_{55}+c_{31}\right)+c_{55}\left(c_{44}+c_{32}\right)-c_{45}\left(2 c_{45}+c_{36}\right),
\end{array}\right\}
$$

and $\psi(x, y)$ satisfies the differential equation

$$
\begin{array}{r}
b_{22} \frac{\partial^{4} \psi}{\partial x^{4}}-3 b_{26} \frac{\partial^{4} \psi}{\partial x^{3} \partial y}+2\left(b_{12}+b_{66}\right) \frac{\partial^{4} \psi}{\partial x^{2} \partial y^{2}}- \\
3 b_{16} \frac{\partial^{4} \psi}{\partial x \partial y^{3}}+b_{11} \frac{\partial^{4} \psi}{\partial y^{4}}=b_{2} \frac{\partial_{2} \phi}{\partial x^{2}}-2 b_{6} \frac{\partial^{2} \phi}{\partial x \partial y}+ \\
b_{1} \frac{\partial^{2} \phi}{\partial y^{2}}+b_{0}
\end{array}
$$

where

$$
b_{i j}=b_{j i}=\left|\begin{array}{l}
c_{i j} c_{i 3} \\
c_{3 j} c_{33}
\end{array}\right| / c_{33} \quad i, j=1,2,6,
$$

and

$$
\begin{aligned}
& b_{i}=c_{i 1} l+c_{i 2} n-c_{i 3}+c_{i 6} m, \quad i=1,2,3,6 . \\
& b_{0}=b_{26} n-b_{16} l .
\end{aligned}
$$

Since both of the functions $\phi(x, y)$ and $\psi(x, y)$ appear in (39), it is apparent that we must first determine $\phi(x, y)$. Fortunately the third boundary condition of the set (17) is independent of $\psi(x, y)$, so that the two boundary value problems are separable.

If the values of $\tau_{y z}$ and $\tau_{z x}$ given by (36) are inserted into the third of (17), we see that this boundary condition will be satisfied if

$$
\begin{array}{r}
\left\{l \frac{\partial \phi}{\partial x}+m \frac{\partial \phi}{\partial y}\right\} \cos (x, \nu)+\left\{m \frac{\partial \phi}{\partial x}+n \frac{\partial \phi}{\partial y}\right\} \cos (y, \nu)= \\
(l y-m x) \cos (x, \nu)+(m y-n x) \cos (y, \nu)
\end{array}
$$

on $C$. It is shown by Sokolnikoff [8, p. 215] that the change of variables

$$
\begin{aligned}
\xi=\sqrt{l n-m^{2}} x, \quad \eta= & y-\frac{m}{l} x, \\
\phi^{\prime}(\xi, \eta) & =\frac{\sqrt{l n-m^{2}}}{l} \phi(\xi, n)
\end{aligned}
$$

will reduce the systems (37) and (41) to the simpler system

$$
\begin{gathered}
\frac{\partial^{2} \phi^{\prime}(\xi, \eta)}{\partial \xi^{2}}+\frac{\partial^{2} \phi^{\prime}(\xi, \eta)}{\partial n^{2}}=0 \\
\frac{d}{d \nu} \phi^{\prime}(\xi, \eta)=\eta \cos (\xi, \nu)-\xi \cos (\eta, \nu)=0 \text { on } C^{\prime} .
\end{gathered}
$$

The problem of determining $\phi(x, y)$ is thus reducible to a problem of Neumann. Since the right hand member of the second of equations (43) is a perfect differential, the determination of $\phi^{\prime}(\xi, \eta)$ is possible, and is unique to within an additive constant.

It should be pointed out that the quantity $\left(l n-m^{2}\right)$ is positive for all elastic media, since from the defining relations (33), we observe that

$$
l n-m^{2} \equiv \frac{c_{33}^{2}}{\beta^{2}}\left(c_{44} c_{55}-c_{45}^{2}\right)
$$

and the positiveness of $\left(c_{14} c_{45}-c_{45}^{2}\right)$ is guaranteed by the positive definite nature of the strain energy form. ${ }^{5}$ Once the function $\phi(x, y)$ has been determined the right-hand member of (39) is a known function.

Let us express the surface tractions $X$ and $Y$ in terms of the tangential traction $T$ and the normal traction $N$, by the relations

$X=N \cos (x, \nu)-T \cos (y, \nu)$,

$$
Y=N \cos (y, \nu)+T \cos (x, \nu) .
$$

${ }_{3}$ See appendix. 
If we make use of the relations (45), the stresses given by (36), and the relations

$$
\cos (x, \nu)=\frac{d y}{d s}, \quad \cos (y, \nu)=-\frac{d x}{d s},
$$

the first two equations of (17) may be rewritten in the form

$$
\begin{aligned}
\frac{d}{d s}\left(\frac{\partial \psi}{d y}\right)= & \left(l \phi+\frac{m}{2} x^{2}-\frac{N}{\alpha}\right) \cos (x, \nu)+ \\
& \left(\frac{n}{2} x^{2}-\frac{l}{2} y^{2}+m \phi+\frac{T}{\alpha}\right) \cos (y, \nu), \\
-\frac{d}{d s}\left(\frac{\partial \psi}{\partial x}\right)= & \left(\frac{n}{2} x^{2}-\frac{l}{2} y^{2}+m \phi-\frac{T}{\alpha}\right) \cos (x, \nu)+ \\
& \left(n \phi-\frac{m}{2} y^{2}-\frac{N}{\alpha}\right) \cos (y, \nu),
\end{aligned}
$$

on $C$. Let us denote the right-hand members of these equations by $F_{2}(x, y)$ and $F_{1}(x, y)$, respectively. If we can find a particular (single-valued) solution, $\psi^{*}(x, y)$ of $(39)$, we must then solve the homogeneous system:

$$
\begin{gathered}
b_{22} \frac{\partial^{4} \psi}{\partial x^{4}}-3 b_{26} \frac{\partial^{4} \psi}{\partial x^{3} \partial y}+2\left(b_{12}+b_{66}\right) \frac{\partial^{4} \psi}{\partial x^{2} \partial y^{2}}- \\
3 b_{16} \frac{\partial^{4} \psi}{\partial x \partial y^{3}}+b_{11} \frac{\partial^{4} \psi}{\partial y^{4}}=0 \\
\frac{d}{d s}\left(\frac{\partial \psi}{\partial y}\right)=-\frac{d}{d s}\left(\frac{\partial \psi^{*}}{\partial y}\right)+F_{2}(x, y) \\
-\frac{d}{d s}\left(\frac{\partial \psi}{\partial x}\right)=\frac{d}{d s}\left(\frac{\partial \psi^{*}}{\partial x}\right)+F_{1}(x, y) .
\end{gathered}
$$

It is easily deduced from the results given by Sokolnik of [7, sections 66 and 95] in his discussion of the theory of anisotropic plates that the system (48) possesses an essentially unique, single-valued solution $\psi(x, y)$, provided that (a) the roots of the characteristic equation

$$
b_{22} \lambda^{4}-3 b_{26} \lambda^{3}+2\left(b_{12}+b_{66}\right) \lambda^{2}-3 b_{16} \lambda+b_{11}=0
$$

are all complex, and (b)

$\int_{C} F_{2} d s=0, \quad \int_{C} F_{1} d s=0, \quad \int_{C}\left(x F_{1}-y F_{2}\right) d s=0$.

It was shown by Lechnitzky ${ }^{6}$ [3] and [4], through energy considerations that the roots of eq (49) are always complex. Thus condition (a) is fulfilled.

We shall test the functions $F_{1}(x, y)$ and $F_{2}(x, y)$, in the first two equations of (50). These equations become

- Lechnitzky considered a similar equation, arising in the study of anisotropic plates, whose roots are the negatives of the roots of eq (49).

$$
\left.\begin{array}{r}
\int_{C}\left(l \phi+\frac{m}{2} x^{2}\right) d y-\left(\frac{n}{2} x^{2}-\frac{l}{2} y^{2}+m \phi\right) d x \\
=\frac{1}{\alpha} \int_{C}\{N \cos (x, \nu)-T \cos (y, \nu)\} d s \\
\int_{C}\left(\frac{n}{2} x^{2}-\frac{l}{2} y^{2}+m \phi\right) d y-\left(n \phi-\frac{m}{2} y^{2}\right) d x \\
=\frac{1}{\alpha} \int_{C} N \cos (y, \nu)+T \cos (x, \nu) d s
\end{array}\right\}
$$

The right-hand members, by the use of eq (45) are seen to represent the components of the resultant force per unit length.

By making use of Green's theorem,

$$
\int_{C} N d y-M d x=\int_{R}\left(\frac{\partial N}{\partial x}+\frac{\partial M}{\partial y}\right) d S,
$$

we rewrite the left-hand members of (51) in the forms

$$
\begin{aligned}
& \int_{R}\left\{l\left(\frac{\partial \phi}{\partial x}-y\right)+m\left(\frac{\partial \phi}{\partial y}+x\right)\right\} d S \\
& \int_{R}\left\{m\left(\frac{\partial \phi}{\partial x}-y\right)+m\left(\frac{\partial \phi}{\partial y}+x\right)\right\} d S .
\end{aligned}
$$

Since $\phi(x, y)$ satisfies the differential equation (37)

$$
l \frac{\partial^{2} \phi}{\partial x^{2}}+2 m \frac{\partial^{2} \phi}{\partial x \partial y}+n \frac{\partial^{2} \phi}{\partial y^{2}}=0,
$$

we may rewrite the expressions (53) in the forms

$$
\begin{gathered}
\int_{R}\left[\frac{\partial}{\partial x}\left\{x l\left(\frac{\partial \phi}{\partial x}-y\right)+x m\left(\frac{\partial \phi}{\partial y}+x\right)\right\}+\right. \\
\left.\frac{\partial}{\partial y}\left\{x m\left(\frac{\partial \phi}{\partial x}-y\right)+x n\left(\frac{\partial \phi}{\partial y}+x\right)\right\}\right] d S \\
\int_{R}\left[\frac{\partial}{\partial y}\left\{y n\left(\frac{\partial \phi}{\partial y}+x\right)+y m\left(\frac{\partial \phi}{\partial x}-y\right)\right\}+\right. \\
\left.\frac{\partial}{\partial x}\left\{y m\left(\frac{\partial \phi}{\partial y}+x\right)+y l\left(\frac{\partial \phi}{\partial x}-y\right)\right\}\right] d S .
\end{gathered}
$$

Once more we use Green's theorem (52), this time on the expressions (54). These now have the forms

$$
\begin{aligned}
& \int_{C} x {\left[\left\{l \frac{\partial \phi}{\partial x}+m \frac{\partial \phi}{\partial y}-l y+m x\right\} \cos (x, \nu)+\right.} \\
&\left.\left\{m \frac{\partial \phi}{\partial x}+n \frac{\partial \phi}{\partial y}-m y+n x\right\} \cos (y, \nu)\right] d s \\
& \int_{C} y\left[\left\{l \frac{\partial \phi}{\partial x}+m \frac{\partial \phi}{\partial y}-l y+m x\right\} \cos (x, \nu)+\right. \\
&\left.\left\{m \frac{\partial \phi}{\partial x}+n \frac{\partial \phi}{\partial y}-m y+n x\right\} \cos (x, \nu)\right] d s
\end{aligned}
$$


The expressions in brackets vanish, by (41), and hence the first two of our integrability equation (50) become, in this case:

$$
\int_{C} X d s=0, \quad \int_{C} Y d s=0 .
$$

These are the conditions which the prescribed components $X$ and $Y$ of the applied stress were assumed to satisfy in section 3 , hence the first two integrability conditions (50) are satisfied.

Let us now consider the third equation of (50). We express $N$ and $T$ in terms of $X$ and $Y$ and see that we must have

$$
\begin{aligned}
\frac{1}{\alpha} \int_{C}( & x Y-y X) d s \\
= & \int_{C}\left\{\frac{n}{2} x^{2} y-\frac{l}{2} y^{3}+m y \phi-x n \phi+\frac{m}{2} x y^{2}\right\} d x \\
& +\left\{\frac{n}{2} x^{3}-\frac{l}{2} x y^{2}+m x \phi-y l_{\phi}-\frac{m}{2} y x^{2}\right\} d y
\end{aligned}
$$

We apply Green's theorem to the right-hand member of $(56)$, to obtain

$$
\begin{array}{r}
\frac{1}{\alpha} \int_{C}(x Y-y X) d s \\
=\int_{R}\left[n x^{2}-2 m x y+l y^{2}+x\left(m \frac{\partial \phi}{\partial x}+n \frac{\partial \phi}{\partial y}\right)\right. \\
\left.-y\left(l \frac{\partial \phi}{\partial x}+m \frac{\partial \phi}{\partial y}\right)\right] d S .
\end{array}
$$

Now, the left-hand member is proportional to the twisting moment per unit length, $M$, that is,

$$
M=\int_{C}(x Y-y X) d s .
$$

Let us denote by $D^{\prime}$ the expression ${ }^{7}$

$$
\begin{array}{r}
D^{\prime}=\int_{R}\left[n x^{2}-2 m x y+l y^{2}+x\left(m \frac{\partial \phi}{\partial x}+n \frac{\partial \phi}{\partial y}\right)-\right. \\
\left.y\left(l \frac{\partial \phi}{\partial x}+m \frac{\partial \phi}{\partial y}\right)\right] d S .
\end{array}
$$

Then, if $\alpha$ is taken as

$$
\alpha=M / D^{\prime},
$$

the third equation of (50) is satisfied, and a singlevalued solution of (48) exists.

We have satisfied the boundary conditions on the lateral surface; next we shall consider the relaxed boundary conditions on the end sections.

On both end sections we require that there be no resultant forces. Hence we require that

'The quantity $D^{\prime}$ is proportional to the Saint-Venant coefficient of torsional rigidity $D$, which arises in the theory of torsion by end couples.

$$
\int_{R} \tau_{z z} d S=0, \quad \int_{R} \tau_{z z} d S=0, \quad \int_{R} \tau_{z y} d S=0
$$

The first of these conditions can be satisfied by adjusting the parameters $A, B$, and $C$. The second and third are easily shown to hold by considering (53) through (55).

On both ends we require that the bending couples, $\stackrel{z}{M_{x}}$ and $\stackrel{z}{M}_{y}$, vanish. These requirements can be met by adjusting the parameters $A, B$ and $C$, so that

$$
\stackrel{2}{M_{x}} \equiv \int_{R} y \tau_{z z} d S=0, \quad \stackrel{2}{M} \equiv-\int_{R} x \tau_{z z} d S=0 .
$$

On the end $z=0$, we require that $\stackrel{z}{M}_{z}=M \cdot h$ and on the end $z=h$, we must have ${ }^{z} M_{z}=0$. The second condition is easily seen to be satisfied since $\tau_{x z}=$ $\tau_{y z}=0$ for $z=\mathrm{h}$.

Since $\stackrel{z}{M}$ for $z=0$ is given by

$$
\begin{aligned}
\stackrel{2}{M}_{z}=h \alpha \int_{R}\{x & {\left[n\left(\frac{\partial \phi}{\partial y}+x\right)+m\left(\frac{\partial \phi}{\partial x}-y\right)\right]-} \\
y & {\left.\left[l\left(\frac{\partial \phi}{\partial x}-y\right)+m\left(\frac{\partial \phi}{\partial y}+x\right)\right]\right\} d S, }
\end{aligned}
$$

and the integrand is equal to the integrand of (57), it follows that

$$
\stackrel{2}{M}_{z}=h \alpha D^{\prime}=h M
$$

Thus the stresses given by (36) can be made to satisfy the boundary conditions (16a) and (17), the conditions of static equilibrium (10) and the compatibility conditions (12) by proper choice of the constants $l, m$, and $n$, and the functions $\phi(x, y)$ and $\psi(x, y)$.

In the next section we shall specialize the equations to the isotropic case, and in section 6 we shall illustrate the use of the method by calculating the stresses in an elliptic cylinder twisted by constant tangential traction.

\section{Isotropic beam}

If the beam is isotropic the differential equations and boundary conditions are considerably simplified. In this case the elastic constants $c_{i j}$ may be expressed in terms of $E$, the Young's modulus, and $\sigma$, the Poisson ratio, as follows.

$$
\left.\begin{array}{l}
c_{11}=c_{22}=c_{33}=\frac{1}{E} \\
c_{23}=c_{32}=c_{21}=c_{13}=c_{13}=c_{31}=-\frac{\sigma}{E} \\
c_{44}=c_{55}=c_{66}=\frac{1+\sigma}{E} \\
c_{16}=c_{61}=c_{26}=c_{62}=c_{36}=c_{63}=c_{45}=c_{54}=0 .
\end{array}\right\}
$$


The elastic constants $l, m, n, b_{i}, b_{i j}$ are given by

$$
\begin{aligned}
l & =n=\frac{1}{2} \\
m & =0 \\
b_{11} & =b_{22}=\left(1-\sigma^{2}\right) / E \\
b_{66} & =(1+\sigma) / E \\
b_{12} & =-\left(\sigma+\sigma^{2}\right) / E \\
b_{16} & =b_{26}=0 \\
b_{1} & =b_{2}=-b_{3}=(1+\sigma) / E \\
b_{6} & =0 .
\end{aligned}
$$

The function $\phi(x, y)$ must satisfy the conditions

$$
\left.\begin{array}{c}
\nabla^{2} \phi(x, y)=0 \\
\frac{d \phi}{d n}=y \cos (x, \nu)-x \cos (y, \nu), \text { on } C
\end{array}\right\}
$$

and $\psi(x, y)$ must satisfy

$$
\left.\begin{array}{c}
\nabla^{4} \psi(x, y)=0 \\
\frac{d}{d s}\left(\frac{\partial \psi}{\partial y}\right)=\left(\frac{\phi}{2}-\frac{N}{\alpha}\right) \cos (x, \nu)+\left(\frac{x^{2}-y^{2}}{4}+\frac{T}{\alpha}\right) \cos (y, \nu) \\
-\frac{d}{d s}\left(\frac{\partial \psi}{\partial x}\right)=\left(\frac{x^{2}-y^{2}}{4}-\frac{T}{\alpha}\right) \cos (x, \nu)+\left(\frac{\phi}{2}-\frac{N}{\alpha}\right) \cos (y, \nu) .
\end{array}\right\}
$$

The torsional rigidity $D^{\prime}$ is calculated from the defining equation

$$
D^{\prime}=\frac{1}{2} \int_{R}\left[x^{2}+y^{2}+x \frac{\partial \psi}{\partial y}-y \frac{\partial \phi}{\partial x}\right] d x d y
$$

and is related to the Saint-Venant coefficient of torsional rigidity $D$ by the relation

$$
D^{\prime}=D(1+\sigma) / E \text {. }
$$

The stress system in this case has the simpler form,

$$
\begin{aligned}
& \frac{1}{\alpha} \tau_{x x}=\frac{\phi}{2}-\frac{\partial^{2} \psi}{\partial y^{2}} \\
& \frac{1}{\alpha} \tau_{y y}=\frac{\phi}{2}-\frac{\partial^{2} \psi}{\partial x^{2}} \\
& \frac{1}{\alpha} \tau_{z z}=-\sigma \nabla^{2} \psi-\phi+(A+B x+C y) \\
& \frac{1}{\alpha} \tau_{y z}=\frac{1}{2}(h-z)\left(\frac{\partial \phi}{\partial y}+x\right)
\end{aligned}
$$

$$
\begin{aligned}
& \frac{1}{\alpha} \tau_{z x}=\frac{1}{2}(h-z)\left(\frac{\partial \phi}{\partial x}-y\right) \\
& \frac{1}{\alpha} \tau_{x y}=\frac{1}{4}\left(x^{2}-y^{2}\right)+\frac{\partial^{2} \psi}{\partial x \partial y},
\end{aligned}
$$

Con.

and the strains are given by

$$
\left.\begin{array}{l}
\frac{1}{\alpha} e_{x x}=\frac{1+\sigma}{2 E} \phi+\frac{\sigma+\sigma^{2}}{E} \frac{\partial^{2} \psi}{\partial x^{2}}-\frac{1-\sigma^{2}}{E} \frac{\partial^{2} \psi}{\partial y^{2}}-\frac{\sigma}{E}(A+B x+C y) \\
\frac{1}{\alpha} e_{y y}=\frac{1+\sigma}{2 E} \phi-\frac{1-\sigma^{2}}{E} \frac{\partial^{2} \psi}{\partial x^{2}}+\frac{\sigma+\sigma^{2}}{E} \frac{\partial^{2} \psi}{\partial y^{2}}-\frac{\sigma}{E}(A+B x+C y) \\
\frac{1}{\alpha} e_{z z}=-\frac{1+\sigma}{E} \phi+\frac{1}{E}(A+B x+C y) \\
\frac{1}{\alpha} e_{y z}=\frac{1+\sigma}{2 E}(h-z)\left(\frac{\partial \psi}{\partial y}+x\right) \\
\frac{1}{\alpha} e_{z x}=\frac{1+\sigma}{2 E}(h-z)\left(\frac{\partial \psi}{\partial x}-y\right) \\
\frac{1}{\alpha} e_{x y}=\frac{1+\sigma}{E} \frac{\partial^{2} \psi}{\partial y \partial x}+\frac{1+\sigma}{4 E}\left(x^{2}-y^{2}\right) .
\end{array}\right\}
$$

\section{Stresses in an elliptic cylinder}

As an illustration of the theory developed in the preceding sections, we shall calculate the stresses in an anisotropic cylinder of elliptic cross section twisted by a constant tangential traction $T$.

Let the equation of the elliptic boundary, $C$, of the cross section of the cylinder be given in the parametric form

$$
x=a \cos \theta, \quad y=b \sin \theta .
$$

Then,

$$
\cos (x, \nu): \cos (y, \nu)=b \cos \theta: a \sin \theta .
$$

Let us set

$$
\phi(x, y)=\sum_{i+j \leq 2} A_{i j} x^{i} y^{j}, \quad i, j=0,1,2 .
$$

and

$$
\psi(x, y)=\sum_{i+j \leq 4} B_{i j} x^{i} y^{j}, \quad i, j=0,1,2,3,4,
$$

and seek to determine the coefficients $A_{i j}$ and $B_{i j}$ in such a way that (37), (39), (41) and (47) are satisfied.

We first calculate $M$ from the defining relation (58),

$$
M=\int_{C}(x Y-y X) d s
$$

If we insert in (76) the expressions for $Y$ and $X$ given by (45), we find that $M$ is given by ${ }^{8}$

${ }^{8}$ Although $N$ is assumed to be zero in this discussion, we include it in eq (77) to show that a constant normal traction produces no twisting moment. 
$M=\int_{C}\{(-y N+x T) \cos (x, \nu)+(y T+x N) \cos (y, \nu)\} d s$.

By making use of Green's theorem (52), we may rewrite (77) in the form

$$
M=2 T \int_{R} d S=2 \pi a b T .
$$

We insert the expression (74) into (37) and (41) and, after some manipulation obtain

$$
\phi(x, y)=\frac{1}{a^{2} n+b^{2} l}\left\{-b^{2} m x^{2}+\left(b^{2} l-a^{2} n\right) x y+a^{2} m y^{2}\right\}
$$

as the solution of the systems (37) and (41).

Using the value of $\phi(x, y)$ given by (79) in the defining equation (59) we find that $\mathrm{D}^{\prime}$ is given by

$$
D^{\prime}=\frac{\pi a^{3} b^{3}\left(n l-m^{2}\right)}{\left(b^{2} l+a^{2} n\right)} .
$$

Hence, from (60), it follows that

$$
\alpha=\frac{2\left(b^{2} l+a^{2} n\right) T}{a^{2} b^{2}\left(n l-m^{2}\right)} .
$$

We next insert the expression (75) for $\psi(x, y)$ into the two equations (47) and after setting $x=a \cos \theta$, $y=b \sin \theta$, and making use of (73), we obtain a pair of polynomials in $\sin \theta$ and $\cos \theta$. These are expanded in Fourier series and the coefficients of the various terms are equated to zero. We thus find that, in order to satisfy the boundary conditions, $\psi(x, y)$ must be given by the expression

$24 a^{2} b^{2}\left(a^{2} n+b^{2} l\right) \cdot \psi(x, y)=b^{4}\left(3 \operatorname{lm} b^{2}-5 m n a^{2}+\gamma\right) x^{4}+$ $4 a^{2} b^{2}\left(b^{2} m^{2}-a^{2} n^{2}\right) x^{3} y+2 a^{2} b^{2} \gamma x^{2} y^{2}$

$+4 a^{2} b^{2}\left(b^{2} l^{2}-a^{2} m^{2}\right) x y^{3}+a^{4}\left(5 l m b^{2}-3 m n a^{2}+\gamma\right) y^{4}-$

$$
\begin{aligned}
& 2 a^{2} b^{4}\left(3 \operatorname{lm} b^{2}-3 m n a^{2}+\gamma\right) x^{2}- \\
& 2 a^{4} b^{2}\left(3 \operatorname{lm} b^{2}-3 m n a^{2}+\gamma\right) y^{2},
\end{aligned}
$$

where $\gamma$ is a constant, as yet arbitrary.

If the value of $\psi(x, y)$ given by (82) is inserted in the differential equation (39), it is found that $\gamma$ must satisfy the relation

$$
\begin{aligned}
\left\{3 b_{22} b^{4}+2 a^{2} b^{2}\left(b_{12}+b_{66}\right)+3 b_{11} a^{4}\right\} \cdot \gamma & \\
= & 3 b_{22} b^{4}\left(5 m n a^{2}-3 l m b^{2}\right)+9 b_{26} a^{2} b^{2}\left(b^{2} m^{2}-a^{2} n^{2}\right)+ \\
& 9 b_{16} a^{2} b^{2}\left(b^{2} l^{2}-a^{2} m^{2}\right)+3 b_{11} a^{4}\left(3 m n a^{2}-5 l m b^{2}\right)- \\
& 6 b_{2} a^{2} b^{4} m+6 b_{6} a^{2} b^{2}\left(a^{2} n-b^{2} l\right)+6 b_{1} a^{4} b^{2} m+ \\
& 3 a^{2} b^{2}\left(a^{2} n+b^{2} l\right)\left(b_{26} n-b_{16} l\right) .
\end{aligned}
$$

Inserting the values of $\phi(x, y), \alpha$, and $\psi(x, y)$ given above into (36) we obtain the stress system

$$
\begin{aligned}
& \frac{\tau_{x x}}{T}=\frac{\left(3 m n a^{2}-3 \operatorname{lm} b^{2}-\gamma\right)\left(b^{2} x^{2}+3 a^{2} y^{2}-a^{2} b^{2}\right)-6 a^{2} b^{2}\left(l n-m^{2}\right) x y}{3 a^{2} b^{4}\left(\ln -m^{2}\right)} \\
& \frac{\tau_{y y}}{T}=\frac{\left(3 m n a^{2}-3 \operatorname{lm} b^{2}-\gamma\right)\left(3 b^{2} x^{2}+a^{2} y^{2}-a^{2} b^{2}\right)+6 a^{2} b^{2}\left(\ln -m^{2}\right) x y}{3 a^{4} b^{2}\left(\ln -m^{2}\right)} \\
& \frac{\tau_{x y}}{T}=\frac{3\left(\ln -m^{2}\right)\left(b^{2} x^{2}-a^{2} y^{2}\right)-2\left(3 m n a^{2}-3 \ln b^{2}-\gamma\right) x y}{3 a^{2} b^{2}\left(\ln -m^{2}\right)} \\
& \frac{\tau_{y z}}{T}=\frac{4 x(h-z)}{a^{2}} \\
& \frac{\tau_{z x}}{T}=-\frac{4 y(h-z)}{b^{2}} \\
& \frac{\tau_{z z}}{T}=p^{\prime} x^{2}+q^{\prime} x y+r^{\prime} y^{2}+A+B x+C y,
\end{aligned}
$$

where

$$
\left.\begin{array}{l}
p^{\prime}=\frac{3 b^{2} c_{32}\left(3 l m b^{2}-5 m n a^{2}+\gamma\right)-3 a^{2} c_{36}\left(b^{2} m^{2}-a^{2} n^{2}\right)+a^{2} c_{31} \gamma}{6 a^{2} c_{33}\left(b^{2} l+a^{2} n\right)}-\frac{c_{31} m+c_{36} n}{c_{33}}+\frac{b^{2} m}{\left(b^{2} l+a^{2} n\right)} \\
q^{\prime}=\frac{3 c_{32}\left(b^{2} m^{2}-a^{2} n^{2}\right)-c_{36} \gamma+3 c_{31}\left(b^{2} l^{2}-a^{2} m^{2}\right)+3 c_{33}\left(a^{2} n-b^{2} l\right)}{3 c_{33}\left(b^{2} l+a^{2} n\right)} \\
r^{\prime}=\frac{b^{2} c_{32} \gamma-3 b^{2} c_{36}\left(b^{2} l^{2}-a^{2} m^{2}\right)+3 a^{2} c_{31}\left(5 l m b^{2}-3 m n a^{2}+\gamma\right)}{6 b^{2} c_{33}\left(b^{2} l+a^{2} n\right)}+\frac{c_{32} m+c_{36} l}{c_{33}}-\frac{a^{2} m}{b^{2} l+a^{2} n} \cdot
\end{array}\right\}
$$


To evaluate the parameters $A, B, C$, in order that the conditions

$$
\int_{R} \tau_{z z} d S=0, \quad \int_{R} x \tau_{z z} d S=0, \quad \int_{R} y \tau_{z z} d S=0
$$

are satisfied we write

$$
\left.\begin{array}{l}
\int_{R}\left(p^{\prime} x^{2}+q^{\prime} x y+r^{\prime} y^{2}+A+B x+C y\right) d x d y=0 \\
\int_{R}\left(p^{\prime} x^{3}+q^{\prime} x^{2} y+r^{\prime} x y^{2}+A x+B x^{2}+C x y\right) d x d y=0 \\
\int_{R}\left(p^{\prime} x^{2} y+q^{\prime} x y^{2}+r^{\prime} y^{3}+A y+B x y+C y^{2}\right) d x d y=0 .
\end{array}\right\}
$$

If we denote by $R, I_{x}$, and $I_{y}$ the area and the moments of inertia of the elliptic section, the system (86) can be written in the form

$$
p^{\prime} I_{y}+r^{\prime} I_{x}+A R=0, \quad B I_{y}=0, \quad C I_{x}=0 .
$$

For the ellipse,

$$
R=\pi a b, \quad I_{x}=\frac{\pi b^{3} a}{4}, \quad I_{y}=\frac{\pi a^{3} b}{4} .
$$

Hence the parameters $A, B$, and $C$ are given by

$$
A=-\frac{a^{2} p^{\prime}+b^{2} r^{\prime}}{4}, \quad B=0, \quad C=0,
$$

and the proposed problem has been completely solved.

For the isotropic elliptic cylinder the solution may be simplified by using the relations given in section 5 .

The solution for this case is given by:

$$
\begin{aligned}
\phi(x, y) & =\frac{b^{2}-a^{2}}{a^{2}+b^{2}} x y \\
D^{\prime} & =\frac{\pi a^{3} b^{3}}{2\left(a^{2}+b^{2}\right)} \\
\alpha & =\frac{4\left(a^{2}+b^{2}\right) T}{a^{2} b^{2}} \\
\gamma & =0 \\
\psi(x, y) & =\frac{b^{2} x y^{3}-a^{2} x^{3} y}{12\left(a^{2}+b^{2}\right)} \\
\frac{\tau_{x x}}{T} & =-\frac{2 x y}{b^{2}} \\
\frac{\tau_{y y}}{T} & =\frac{2 x y}{a^{2}} \\
\frac{\tau_{x y}}{T} & =\frac{b^{2} x^{2}-a^{2} y^{2}}{a^{2} b^{2}}
\end{aligned}
$$

$$
\begin{aligned}
& \frac{\tau_{y z}}{T}=\frac{4 x(h-z)}{a^{2}} \\
& \frac{\tau_{z x}}{T}=-\frac{4 y(h-z)}{b^{2}} \\
& \frac{\tau_{z z}}{T}=\frac{2 \sigma\left(a^{2}-b^{2}\right) x y}{a^{2} b^{2}} .
\end{aligned}
$$

Con.

For an isotropic circular cylinder of radius $a$ the results are given by

$$
\begin{aligned}
\phi(x, y) & =0 \\
D^{\prime} & =\frac{\pi a^{4}}{4} \\
\alpha & =\frac{8 T}{a^{2}} \\
\psi(x, y) & =\frac{x y^{3}-x^{3} y}{24} \\
\frac{\tau_{x x}}{T} & =\frac{2 x y}{a^{2}} \\
\frac{\tau_{y y}}{T} & =\frac{2 x y}{a^{2}} \\
\frac{\tau_{x y}}{T} & =\frac{x^{2}-y^{2}}{a^{2}} \\
\frac{\tau_{y z}}{T} & =\frac{4 x(h-z)}{a^{2}} \\
\frac{\tau_{z x}}{T} & =-\frac{4 y(h-z)}{a^{2}} \\
\frac{\tau_{z z}}{T} & =0 .
\end{aligned}
$$

\section{Methods for obtaining solutions of the boundary-value problems}

The determination of the stresses and displacements in the twisted cylinder has been made to depend upon the solution of the two linear partial differential equations,

$$
l \frac{\partial^{2} \phi}{\partial x^{2}}+2 m \frac{\partial^{2} \phi}{\partial x \partial y}+n \frac{\partial^{2} \phi}{\partial y^{2}}=0
$$

and

$$
\begin{gathered}
b_{22} \frac{\partial^{4} \psi}{\partial x^{4}}-3 b_{26} \frac{\partial^{4} \psi}{\partial x^{3} \partial y}+2\left(b_{12}+b_{66}\right) \frac{\partial^{4} \psi}{\partial x^{2} \partial y^{2}}-3 b_{16} \frac{\partial^{4} \psi}{\partial x \partial y^{3}}+ \\
b_{11} \frac{\partial^{4} \psi}{\partial y^{4}}=b_{2} \frac{\partial^{2} \phi}{\partial x^{2}}-2 b_{6} \frac{\partial^{2} \phi}{\partial x \partial y}+b_{1} \frac{\partial^{2} \phi}{\partial y^{2}}+b_{0},
\end{gathered}
$$


together with the appropriate boundary conditions, (41) and (47).

It was shown in section 4 that (92) can, by a simple change of variables, be transformed into the form

$$
\nabla^{2} \phi(x, y)=0,
$$

and the various methods of potential theory are available for the solution of this problem of Neumann. An elegant method of solution of the Neumann problem, due to Muschelišvili, is discussed in Sokolnikoff [8, p. 170].

The determination of the function $\psi(x, y)$ is considerably more difficult. An equation similar to (93) arises in the theory of anisotropic plates and a great deal of work has been done on the problem of solving this equation. A series of papers by Green and Taylor [2] gives a method of solution of the system (93)-(47) for the special case (corresponding to the orthotropic beam), where

$$
b_{26}=b_{16}=0,
$$

A perturbation method of solving the system (93)(47), which consists in expressing the solution as a series of biharmonic functions, is described by Sokolnikoff [9]. Another, more elegant method, essentially due to Muschelišvili, depending on conformal mapping is also described by Sokolnikoff $[7$, p. 375] and [10].

If the beam is isotropic, (93) has the simple form

$$
\nabla^{4} \psi(x, y)=0,
$$

and the solution is easily obtained in the form

$$
\psi(x, y)=\boldsymbol{R}[f(z)+\bar{z} g(z)],
$$

where $f(z)$ and $g(z)$ are analytic functions of the complex variable $z[\equiv x+i y]$, which are chosen so that the function $\psi(x, y)$ given by eq (97) will satisfy the boundary conditions (47) [10].

\section{Summary}

The determination of the state of stress and the displacements in a built-in anisotropic cylinder twisted by forces uniformly applied along the lateral surface has been made to depend on the solution of two boundary-value problems.

The existence of the solutions of the boundaryvalue problems has been demonstrated, and several methods of solution of the boundary-value problems are indicated.

As an illustration of the method, the stresses in an anistropic beam of elliptic cross section twisted by constant tangential traction were obtained by assuming that the stress functions were second- and fourthdegree polynomials.
In a succeeding paper it is planned to extend the theory to the case where the applied surface tractions vary along the length of the cylinder, that is, where the boundary conditions are given in the form. ${ }^{9}$

$$
\left.\begin{array}{l}
X=f(z) X(s) \\
Y=g(z) Y(s) \quad \text { on } C . \\
Z=0 .
\end{array}\right\}
$$

It is hoped that the theory can be further extended to the problems of extension, bending, and flexure of anisotropic cylindrical beams by forces distributed on the lateral surface. ${ }^{10}$

The boundary conditions for these problems are: (a) Extension:

$$
\begin{array}{ll}
X=0 & \text { on } C \\
Y=0 & \text { on } C,
\end{array}
$$

and $Z$ is prescribed on $C$ subject to the conditions

$$
\int_{C} Z d s \neq 0, \quad \int_{C} x Z d s=0, \quad \int_{C} y Z d s=0 .
$$

(b) Bending:

$$
\begin{array}{ll}
X=0 & \text { on } C \\
Y=0 & \text { on } \mathrm{C},
\end{array}
$$

and $Z$ is prescribed on $C$ subject to the conditions

$$
\int_{C} Z d s=0, \quad \int_{C} x Z d s \neq 0, \quad \int_{C} y Z d s \neq 0 .
$$

(c) Flexure:

$$
T=0 \quad \text { on } C,
$$

and $X$ and $Y$ are prescribed on $C$ subject to the conditions

$$
\int_{C} X d s \neq 0, \quad \int_{C} Y d s \neq 0, \quad \int_{C}(x Y-y X) d s=0 .
$$

It is apparent that a superposition of solutions corresponding to the boundary conditions (98) through (101) can be made to satisfy quite general boundary conditions on the lateral surface.

\footnotetext{
$\because$ Zwolinsky and Riz [12] treated the case (for isotropic cylinders) where $f(z) \equiv z$, $N=0, T=$ constant on $C$.

10 The problems of extension, bending, and flexure of isotropic (and orthotropic) cylinders by forces applied on the end sections have been solved by Saint-Venant [8 p. 100].
} 


\section{References}

[1] L. N. G. Filon, Phil. Trans. Roy. Soc. [A] 198, 147 (1902).

[2] A. E. Green and G. I. Taylor, Stress systems in aeolotropic plates, Proc. Roy. Soc. London, Parts I and II 173, 162, 173 (1939); Parts III, V, VI, VII, 184, 181, $231,289,301$ (1945).

[3] S. G. Lechnitzky, USSR Academy of Sciences, J. Applied Math. Mech., new series 2, 345 to 368 (1939)

[4] S. G. Lechnitzky, USSR Academy of Sciences, J. Applied Math. Mech., new series 6, 3 to 18 (1942)

[5] A. E. H. Love, A treatise on the mathematical theory of elasticity (Dover Publications, New York, N. Y., 1944).

[6] P. M. Riz, USSR Academy of Sciences, J. Applied Math. Mech., new series 4, no. 2, 121 to 122 (1940).

[7] I. S. Sokolnikoff, Mathematical theory of elasticity (Brown Univ. Press, 1941)

[8] I. S. Sokolnikoff, Mathematical theory of elasticity (McGraw-Hill Book Co., Inc., New York, N. Y., 1946).

[9] I. S. Sokolnikoff, article in the Proceedings of the Third Symposium in Applied Mathematics (American Mathematical Society, 1950).

[10] I. S. Sokolnikoff, Some new methods of solution of two dimensional problems in elasticity, Bull. AMS 48, 539 to $555(1942)$.

[11] A. Timpe, Math. Ann. 71, 480 (1912).

[12] N. V. Zwolinsky and P. M. Riz, Bul. de l'acad. des sci. de l'URSS, class. des sci. math. et nat., no. 10, 21 to $26(1939)$.

\section{Appendix. Strain Energy and Elastic Symmetry}

The strain energy, $W$, of a strained elastic medium is defined by $[8$, p. 88]

$$
2 W=\tau_{x x} e_{x x}+\tau_{y y} e_{y y}+\tau_{z z} e_{z z}+2 \tau_{y z} e_{y z}+2 \tau_{z x} e_{z x}+2 \tau_{x y} e_{y x} .
$$

In the linear theory of elasticity the strain energy is assumed to be given also by a positive definite quadratic form in the components of the strain tensor by

$$
\left.\begin{array}{rl}
W=\frac{1}{2} b_{11} e_{x x}^{2}+b_{12} e_{x x} e_{y y}+b_{13} e_{x x} e_{z z} & +b_{14} e_{x x} e_{y z}+b_{15} e_{x x} e_{z x}+b_{16} e_{x x} e_{x y} \\
+\frac{1}{2} b_{22} e_{y y}^{2}+b_{23} e_{y y} e_{z z}+b_{24} e_{y y} e_{y z}+b_{25} e_{y y} e_{z x}+b_{26} e_{y y} e_{x y} \\
+\frac{1}{2} b_{33} e_{z x}^{2}+b_{34} e_{z z} e_{y z}+b_{35} e_{z z} e_{z x}+b_{36} e_{z z} e_{x y} \\
+\frac{1}{2} b_{44} e_{y z}^{2}+b_{45} e_{y z} e_{z x}+b_{46} e_{y z} e_{x y} \\
+\frac{1}{2} b_{55} e_{z x}^{2}+b_{56} e_{z x} e_{x y} \\
+\frac{1}{2} b_{66} e_{x y}^{2}
\end{array}\right)
$$

The positive definite nature of the form (103) imposes certain requirements on the signs of the elements of the matrix

$$
\left(\begin{array}{llllll}
b_{11} & b_{12} & b_{13} & b_{14} & b_{15} & b_{16} \\
b_{12} & b_{22} & b_{23} & b_{24} & b_{25} & b_{26} \\
b_{13} & b_{23} & b_{33} & b_{34} & b_{35} & b_{36} \\
b_{14} & b_{24} & b_{34} & b_{44} & b_{45} & b_{46} \\
b_{15} & b_{25} & b_{35} & b_{45} & b_{55} & b_{56} \\
b_{16} & b_{56} & b_{36} & b_{46} & b_{56} & b_{66}
\end{array}\right)
$$

An elastic medium is said to possess elastic symmetry in a plane if a reflection of coordinates in that plane leaves the strain energy form (103) invariant. Let us consider elastic symmetry in the $x y$-plane. The form (103) must be invariant under the transformation

$$
\left.\begin{array}{l}
x^{\prime}=x \\
y^{\prime}=y \\
z^{\prime}=-z .
\end{array}\right\}
$$

The components of the strain tensor transform according to the relations

$$
\left.\begin{array}{l}
e_{x^{\prime} x^{\prime}}=e_{x x}, \quad e_{y^{\prime} y^{\prime}}=e_{y y}, \quad e_{z^{\prime} z^{\prime}}=e_{z y} \\
e_{y^{\prime} z^{\prime}}=-e_{y z}, \quad e_{z^{\prime} x^{\prime}}=-e_{z x}, \quad e_{x^{\prime} y^{\prime}}=e_{x y},
\end{array}\right\}
$$

and if the form (103) is to be invariant under the transformation, we must have

$$
b_{14}=b_{15}=b_{24}=b_{25}=b_{35}=b_{46}=b_{56}=0 .
$$

The matrix (104) then has the form

$$
\left(\begin{array}{llllll}
b_{11} & b_{12} & b_{13} & 0 & 0 & b_{16} \\
b_{12} & b_{22} & b_{23} & 0 & 0 & b_{26} \\
b_{13} & b_{23} & b_{33} & 0 & 0 & b_{36} \\
0 & 0 & 0 & b_{44} & b_{45} & 0 \\
0 & 0 & 0 & b_{45} & b_{55} & 0 \\
b_{16} & b_{26} & b_{36} & 0 & 0 & b_{66} .
\end{array}\right)
$$

Since the matrix (104a) is obtained from a positive definite quadratic form it follows that

$$
\left|\begin{array}{ll}
b_{44} & b_{45} \\
b_{45} & b_{55}
\end{array}\right|>0
$$

By differentiating (102) and (103) with respect to $e_{x x}$, and comparing, Hooke's law is obtained, expressing the stresses in terms of the strains.

If this expression of Hooke's law is solved for the strains in terms of stresses and the coefficients are denoted by $c_{11}, \ldots, c_{66}$ as in section 2 , it will be seen that the determinant

$$
\left|\begin{array}{cc}
c_{44} & c_{45} \\
c_{45} & c_{55}
\end{array}\right|
$$

is a positive multiple of the determinant in (108), and hence is positive, as asserted in section 4 .

Los Angeles, May 26, 1951. 